\title{
Missed Odontoid Fracture Associated to Atlas fracture and Atlanto-Axial Subluxation in a Body Builder. A Brief Technical Review
}

ISSN: 2637-7748

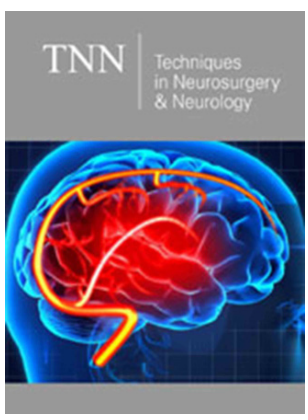

*Corresponding author: Juan F Martínez Canca, Consultant Neurosurgeon, NeuroKonsilia

Submission: 毕 April 14, 2021

Published: 泚 May 5, 2021

Volume 4 - Issue 2

How to cite this article: Pablo Martínez Soler, Juan F Martínez Canca. Missed Odontoid Fracture Associated to Atlas fracture and Atlanto-Axial Subluxation in a Body Builder. A Brief Technical Review. Tech Neurosurg Neurol. 4(2). TNN. 000584. 2021.

DOI: 10.31031/TNN.2021.04.000584

Copyright@ Juan F Martínez Canca, This article is distributed under the terms of the Creative Commons Attribution 4.0 International License, which permits unrestricted use and redistribution provided that the original author and source are credited.

\author{
Pablo Martínez Soler ${ }^{1}$ and Juan F Martínez Canca ${ }^{2 *}$ \\ ${ }^{1}$ Medical Student University of Aberdeen, Scotland \\ ${ }^{2}$ Consultant Neurosurgeon, NeuroKonsilia, Spain
}

\begin{abstract}
We present the case of a healthy 37-year-old male body builder champion who sustained a significant neck injury after diving into a shallow swimming pool whilst intoxicated during his holiday abroad. His severe cervical injuries were astoundingly missed at the local A\&E Department and he was discharged to fly back home on a soft collar and analgesics. He presented to his General Practitioner two weeks later complaining of refractory neck pain and significant neurological signs and symptoms. Following spinal multidisciplinary discussion at the local specialised Unit, halo vest immobilisation was identified as the best treatment option. A 24-week period was required to achieve complete healing of the injuries and full return to normal functionality.
\end{abstract}

\section{Introduction}

Significant force is required to sustain an odontoid fracture, especially in young healthy individuals. Common scenarios for this situation are road traffic accidents, sport accidents, and falls from a height. Population above the age of 75 years can suffer this type of injury after simple ground level falls or minor to moderate neck axial loadings. Pathological fractures are also a possible aetiology, although much less frequently than the former. Flexion injury is the most common mechanism and is normally associated to anterior C1-C2 displacement (atlanto-axial subluxation). Conversely, extension injuries are less likely to produce odontoid fractures and they are usually linked to posterior displacement. Although there are no conclusive studies regarding mortality at the time of the injury, it is estimated that $25-40 \%$ these injuries will lead to immediate fatality. Presenting symptoms include a wide spectrum which ranges from minor deficits such as neck and scalp paraesthesia to monoparesis and quadriplegia. As a general guide to understand these fractures, the Anderson and D'Alonzo classification has been used since its successful publication in 1974. This study classifies fractures in groups I, II, IIA, and III according to the morphological features, and establishes the anatomical stability of each group (Figure 1). Type I fractures are deemed to be unstable, types II and IIA are considered 'usually' unstable, and type III fractures are 'usually' stable [1-6].

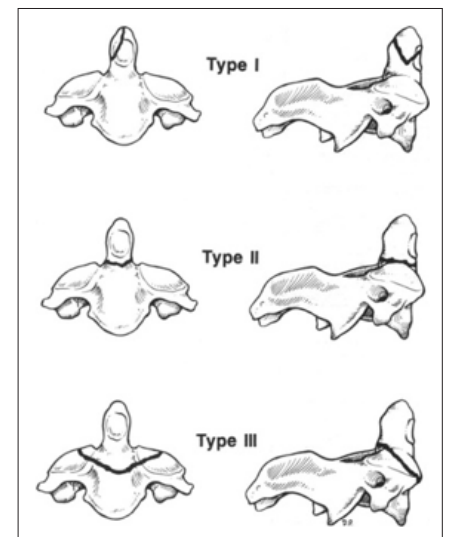

Figure 1: 
When associated with $\mathrm{C} 1$ fractures and/or C1-C2 subluxations, the diagnosis of the clinical picture must pay very special attention in determining whether there is associated ligamental instability. To this aim, the Rule of Spence (published in 1970) is a radiographic method that attempts to determine the integrity of the transverse ligament when obtaining an 'open mouth' (odontoid view) radiograph (Figure 2). The outer edges of $\mathrm{C} 1$ lateral masses should not extend over (or "hang over") the outer edge of C2 superior articular facets. If there is such overhang, the degree should be measured on both sides and, if the combined distance exceeds more than $6.9 \mathrm{~mm}$, then there should be concern for a possible transverse ligament injury and the patient should get an MRI and flexion/ extension imaging under medical supervision. Treatment remains controversial since there has been no agreement in identifying the factors that will predict which type II odontoid fractures will be most likely to heal with immobilisation and which ones will require surgical input. Having reviewed the available literature, surgical treatment is advised for odontoid type II fractures in patients aged 7 years and beyond with any of the following:
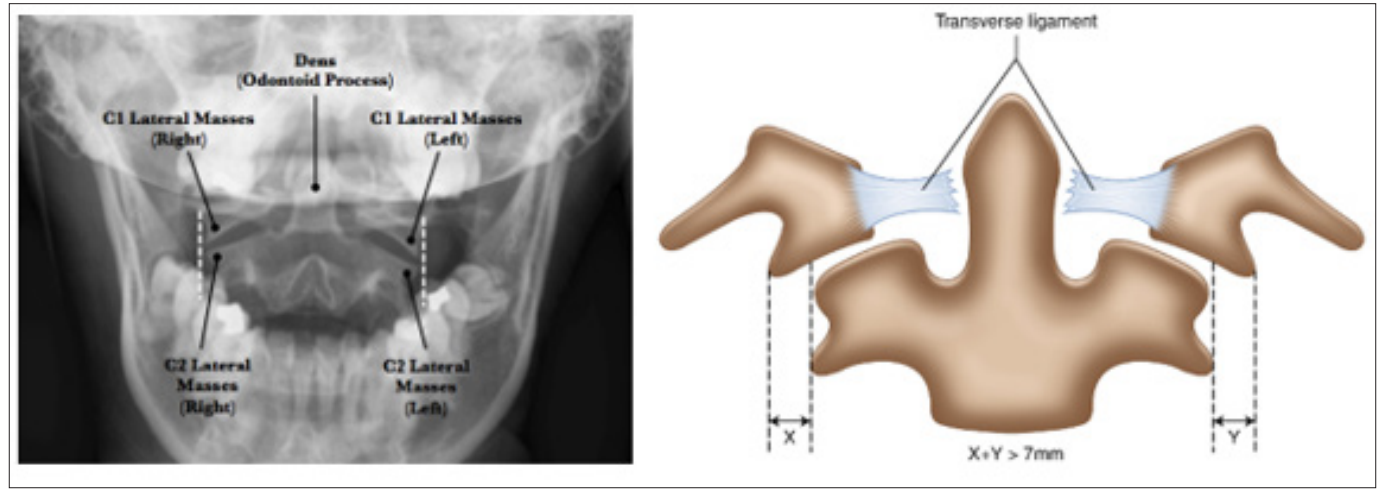

\section{Figure 2:}

a) Displacement greater than $5 \mathrm{~mm}$.

b) Instability at the fracture site after implementation of the halo vest.

c) Patients older than 50 years of age (dramatic increase in the non-union rate).

d) Non-union (especially if myelopathy is present).

e) Disruption of the transverse ligament (associated with delayed instability).

The 'broad guidelines' for treatment of these fractures set the following criteria:

i. Isolated type II odontoid fracture in adults aged 50 years and beyond should be considered for surgical stabilisation and fusion.

ii. Types I, II, and III fractures may be managed initially with external cervical immobilisation.

iii. Types II and III: consider surgical fixation for:

a) Fracture displacement equal or greater than $5 \mathrm{~mm}$.

b) Type IIA fracture (the comminute type).

c) Inability to maintain alignment with external immobilisation.

Surgical options include odontoid compression screw and C1C2 arthrodesis (wiring, fusion, trans articular screw fixation, Halifax clamps, etc). Immobilisation is recommended for those cases not meeting the surgical criteria. The minimum period of treatment is 10-12 weeks. Options include halo vest, rigid collar, SOMI brace, and other rigid cervical orthosis. Atlas fractures can be classified in three main groups:

Type I: fractures involving a single arch (31 to 45\%).

Type II: burst fracture (37 to 51\%) known as Jefferson fracture.

Type III: lateral mass fractures (13 to $37 \%$ ).

Historical interest is due to Jefferson fracture, described in 1920 by Sir Geoffrey Jefferson as a four-point burst fracture of C1 ring, but the term is currently more often used to include the (more frequent) three-point or two-point fractures, the latter through the $\mathrm{C} 1$ arch which is the thinnest portion. Most common aetiology is excessive axial loading (also called "blow out fracture"). There is an associated risk of C2 fracture in $41 \%$ of the cases. This fracture is deemed to be unstable and, characteristically, with no neurological deficit if isolated, due to the large spinal canal diameter at this level as well as the tendency for fragments to be forced outwards away from the spinal cord. It is important to remember that stability of the occipito-atlanto-axial complex is primarily due to ligaments, with marginal contribution from bony structures or articulations. Integrity of Transverse Atlantal Ligament (TAL) is the most important determinant of stability in these fractures, hence the crucial importance of performing MRI evaluation along with primary radiological assessment of the area, including complete C-spine series and thin cut high resolution CT from C1 to C3. Surgical treatment depends almost entirely of the integrity of the Transverse Atlantal ligament. Although immobilisation (in several forms) is statistically the commonest of the treatments, surgical options include different types of arthrodesis (e.g. occipito-cervical fusion, posterior C1 screw placement, anterior transoral screw/ plate placement) [6-9]. 


\section{Case Report}

A previously fit and healthy 37-year-old right-handed male was taken to the very busy local ED in the small hours, following a head injury sustained after diving into the shallow end of a swimming pool whilst intoxicated at a party during the last night of his holiday abroad. Overall general condition was stable, and the patient reported no significant symptoms other than slurred speech assumed to be linked to excessive alcohol intake at the time. Physical and neurological assessment revealed a large forehead contusion with a $4 \mathrm{~cm}$ laceration that was bleeding, and tingling affecting the right hand and forearm. Rotation of his neck was slightly restricted due to pain, but the assessing ED physician documented that this could be due to his "muscle bulk" as the patient was a long-term body builder champion. Following this, cervical spine radiographic evaluation was performed (AP and lateral views) and the patient was discharged on conventional analgesics and the advice of wearing a soft cervical collar for comfort, in view of his return to the UK on a commercial flight next morning. The established diagnosis was "mild head injury secondary to alcohol intoxication". Upon return to his home the patient removed the cervical collar after 24 hours and resumed his sport activity routines at the gym which included long hours of regular heavy lifting and extreme physical maintenance program in preparation for a forthcoming body building competition. However, he noticed intense pain in the cranio-cervical junction which restricted his range of mobility as well as progressive weakness in both arms along with a sensation of permanent numbness in his right forearm and hand that gradually appeared contralaterally. Self-prescribed medication consisted of regular over-the-counter Paracetamol and Ibuprofen. It was only after two further weeks of continuous and increasing trouble when he visited his General Practitioner reporting ineffectiveness of the medication and spiralling symptoms, especially noticeable during his weight-lifting routines as he could not feel the weight handles and experienced constant electric shocks along his body that made impossible to complete his sessions safely. Physical and neurological evaluation revealed marked paraesthesiae in both hands and right forearm, generalised weakness of all muscle groups in both upper limbs and very restricted neck rotation due to severe muscle contracture. The rest of the assessment was unremarkable. In view of the above, and upon enquiring about the full details of the original episode during his holiday, his General Practitioner implemented immediate spinal board immobilisation at the Surgery and called emergency transport in order to take the patient to the nearest A\&E facility.

Diagnostic investigations performed at the nearby Hospital revealed a type IIA odontoid fracture in the context of Atlas fracture and atlanto-axial dislocation with a C1-C2 'hang over' of $9 \mathrm{~mm}$ (Figure 3). The patient was admitted to the Spinal Unit and the case discussed at the Spinal Multidisciplinary meeting. In view of the findings described, it was decided to implement treatment with halo-vest immobilisation for an initial period of twelve weeks. Outcome: Following the initial immobilisation period, additional radiological follow-up images performed revealed non-union of the fragments, reason for which the immobilization was extended with periodic re-evaluations every two weeks, before deciding upon a possible surgical offer, should permanent failure persist. Eventually, fusion evidence started to appear, and after twelve further weeks the situation granted enough radiological stability to allow removal of the immobilisation device. The patient started a medically supervised physiotherapy and rehabilitation program and gradually resumed normal functionality including sport activity without any specific restriction [9-13].

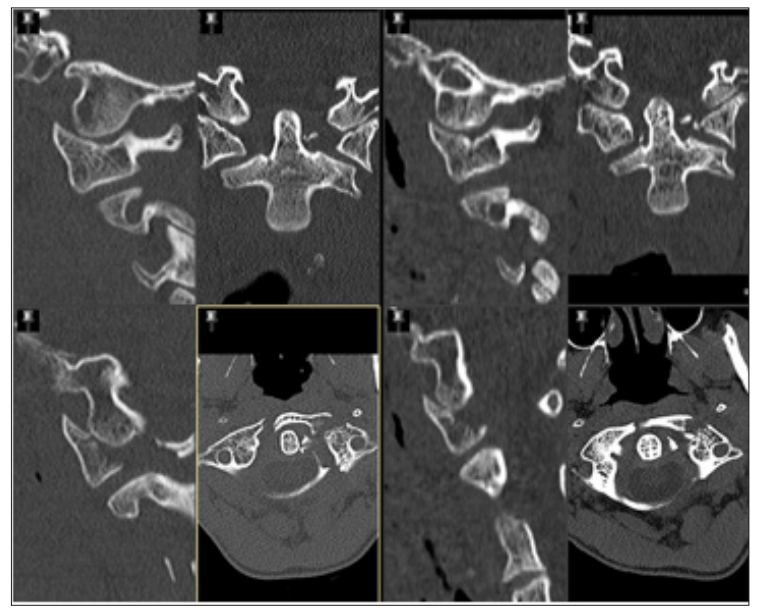

Figure 3:

\section{Discussion}

This case illustrates multiple aspects of the diagnosis (actually, misdiagnosis) and subsequent adequate management of the C1-C2 fracture/dislocation scenario described. The fact that the local ED physician missed this potentially lethal injury and simply discharged the patient on a soft collar with the bare aid of conventional analgesics triggered a series of shocking events that could have been seriously deleterious for the patient's health, for instance, flying back home in a commercial flight without appropriate spinal precautions less than 24 hours after sustaining a cervical injury or resuming high demanding weight lifting activity as part of his gym routines, being totally unaware of his severe underlying condition. Incredibly, even when a dramatically unstable complex fracture existed, the patient continued his physical schedule. Astonishingly, not only he did not damage his neurological structures any further, but was able to over-exercise at the highest level for an additional two weeks before reporting to his General Practitioner. Fortunately, the fast and crucial intervention of his family Doctor who perfectly identified the impending danger after making the right enquiries and putting together essential clinical details provided the correct path for further specialized treatment at the local Spinal Unit.

\section{References}

1. Jefferson G (1920) Fractures of the atlas vertebra: report of four cases, and review of those previously recorded. Br J Surg 7(27): 407-422.

2. Spence KF, Decker S, Sell KW (1970) Bursting atlantal fracture associated with rupture of the transverse ligament. J Bone Joint Surg 52(3): 543549 .

3. Anderson LD, Alonzo RT (1974) Fractures of the odontoid process of the axis. J Bone Joint Surg Am 56(8): 1663-1674. 
4. Papadopoulos SM (1993) Biomechanics of occipito-atlanto-axial trauma. In: Rea GL \& Miller CA (Eds.), Spinal Trauma: Current evaluation and management. Neurosurgical topics, American Association of Neurological Surgeons. USA, pp. 17-23.

5. Ivancic P (2014) Odontoid fracture biomechanics. Spine 39(24): E1403-E1410.

6. Min W, Kim J (2015) Hyperextension injury of the C1-C2 cervical spine with neurologic deficits: horizontal splitting fracture of the $\mathrm{C} 1 \mathrm{arch}$. The Spine Journal 15(2): 370-372.

7. Howard J, Plessis S (2004) Posterolateral dislocation of the C1-C2 articulation associated with fracture of the anterior arch of C1: A case report. Spine 29(24): E562-E564.

8. Naeem A, Alrumaihi G, Bailey C (2016) P136 Type III odontoid fracture with $\mathrm{C} 1$ and $\mathrm{C} 2$ distraction injury manifesting as a variant of occipitalcervical dissociation. Canadian Journal of Neurological Sciences 43(S2): S51-S52.
9. Lefaucheur R, Derrey S, Fetter D, Wallon D, Gilard V (2014) Nontraumatic cervical C1-C2 subluxation. Joint Bone Spine 81(6): 545-546.

10. Hadithy N, Khan A, Banerjee A (2011) A potentially missed cervical (C2) spine fracture. Case Reports 2: bcr1220103576-bcr1220103576.

11. Clasper J, Pailthorpe C (1995) Delayed diagnosis of an odontoid process fracture in an infant. Injury 26(4): 281-282.

12. Riouallon G, Pascal MH (2014) Atlanto-axial dislocation complicating a type II odontoid fracture. Reduction and final fixation. Orthop Traumatol Surg Res 100(3): 341-345.

13. Ross M, Cheeks J (2008) Clinical decision making associated with an undetected odontoid fracture in an older individual referred to physical therapy for the treatment of neck pain. J Orthop Sports Phys Ther 38(7): 418-424.

For possible submissions Click below: 